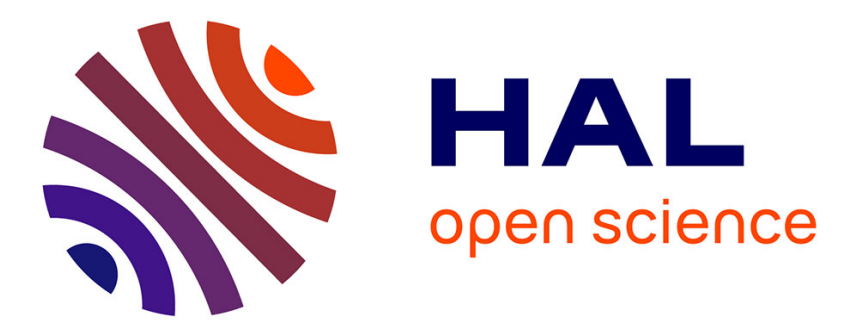

\title{
Probing submillimeter dynamics to access static shear elasticity from polymer melts to molecular fluids
}

\author{
Laurence Noirez
}

\section{To cite this version:}

Laurence Noirez. Probing submillimeter dynamics to access static shear elasticity from polymer melts to molecular fluids. S. Palsule. Polymers and Polymeric Composites: A Reference Series., Springer-verlag, pp.249-271, 2019, Liquid Crystalline Polymers, 10.1007/978-3-642-37179-0_54-1. hal-02355264

\section{HAL Id: hal-02355264 \\ https://hal.science/hal-02355264}

Submitted on 8 Nov 2019

HAL is a multi-disciplinary open access archive for the deposit and dissemination of scientific research documents, whether they are published or not. The documents may come from teaching and research institutions in France or abroad, or from public or private research centers.
L'archive ouverte pluridisciplinaire HAL, est destinée au dépôt et à la diffusion de documents scientifiques de niveau recherche, publiés ou non, émanant des établissements d'enseignement et de recherche français ou étrangers, des laboratoires publics ou privés. 


\title{
Probing Submillimeter Dynamics to Access Static Shear Elasticity from Polymer Melts to Molecular Fluids
}

\author{
Laurence Noirez
}

\section{Contents}

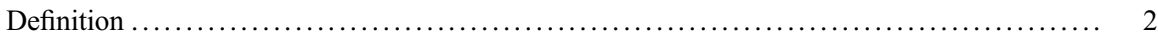

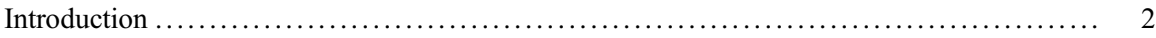

Empirical Origins of the Viscoelastic Approach in Polymer Dynamics and Limitations ...... 4

Hidden Experimental Difficulties Inherent to a Viscoelastic Measurement and to the

Determination of a Viscoelastic Time ........................................ 6

Optimizing the Stress Transmission in Viscoelastic Measurements and Scanning

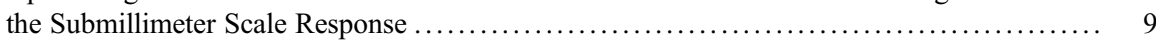

From Surface-Induced Solidification to the Identification of the Submillimeter

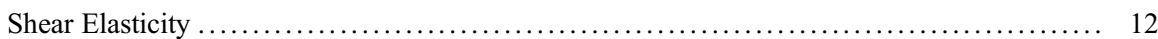

Generalization of the Submillimeter Shear Elasticity to Fluids and Liquids ............... 13

Shear Elasticity and Surface Tension ......................................... 14

Relationships Between Low-Frequency Shear Elasticity and Conventional Viscoelasticity ... 15

Conclusions ............................................................... 19

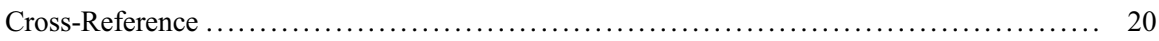

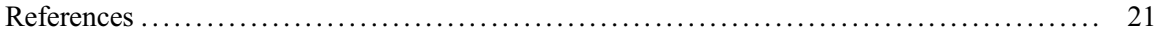

\begin{abstract}
At the millimeter scale and above, liquids and viscoelastic liquids are characterized by an absence of shear elasticity at low frequency $(\sim \mathrm{Hz})$ in contrast to solids or plastic fluids that need to exceed a stress threshold to flow. Below the millimeter scale, the dynamic response exhibits viscoelastic moduli much higher than those measured at larger scale and reveals that fluids possess finite shear elasticity at low frequency. The low-frequency shear elasticity is identified on unentangled and entangled polymers away from the glass transition, molecular glass formers, alkanes, and H-bond liquids, from several tenths to hundredths of
\end{abstract}

L. Noirez ( $\square)$

Laboratoire Léon Brillouin (CEA-CNRS), Université Paris-Saclay, CEA-Saclay, Gif-sur-Yvette Cédex, France

e-mail: laurence.noirez@cea.fr 
millimeter scales. It indicates that liquid molecules are long-range elastically correlated Consequently the thermal and density fluctuations are also elastically correlated, highlighting on the liquid state new mechanisms to consider to understand the microfluidic scale. How to conciliate the low frequency shear elasticity and viscoelasticity theory when the scale goes down to the submillimeter scale? The polymer viscoelasticity theory is founded on the predominance of the molecular dynamics (major intrachain contribution). In contrast, the dynamics of simple liquids is governed by intermolecular forces. How to conciliate intra- versus intermolecular interactions when the polymer weight decreases down to simple liquids whose dynamics are governed by intramolecular interactions? What are the underlying assumptions and their limitations? This entry traces a brief history of the foundations of the viscoelasticity theory, its empirical origin, and presents new developments revealing that the conventional viscoelastic and viscous behaviors might be the asymptotic part of a much broader dynamic response of the liquid state.

\section{Keywords}

Microfluidic · Unentangled and entangled polymer melts · Molecular liquids · Physiologic fluids $\cdot$ Low frequency $\cdot$ Solid-like properties

\section{Definition}

Dynamic mechanical relaxation aims at probing the response of a fluid to a mechanical stress by transmitting a shear stress via fluid/substrate contact in conditions as close as possible to the equilibrium (linear conditions). The characteristic dynamic variables are the viscoelastic moduli (shear elastic and viscous moduli). The dynamic profile is built by varying the frequency in a range typically between $10^{-3}$ and $10^{3} \mathrm{~Hz}$. Dynamic relaxation probes the very low-frequency properties, while the majority of dynamic analysis techniques accesses $\mathrm{MHz}$ and $\mathrm{GHz}$ frequencies (NMR, inelastic scattering, Raman, Brillouin scattering, etc.).

The reliability of the dynamic relaxation measurement is entirely dependent on the quality of the transmission of the stress via the sample via the fluid/substrate interfacial forces (Fig. 1). This is the weak point of the technique. This entry shows that the protocol of dynamic relaxation is improvable by optimizing the interaction conditions between the sample and the substrate. Better measurement performances are obtained in rheometry leading to the demonstration of elastic properties in the liquid state previously neglected.

\section{Introduction}

Progresses in the dynamic characterization of fluids, polymer melts, or solutions are essential given their implementation and the importance of their use in optimal conditions. Among the three states of matter, the liquid state is certainly the most poorly known, hardly modelled, and predictable. Rheology with the emergence of 


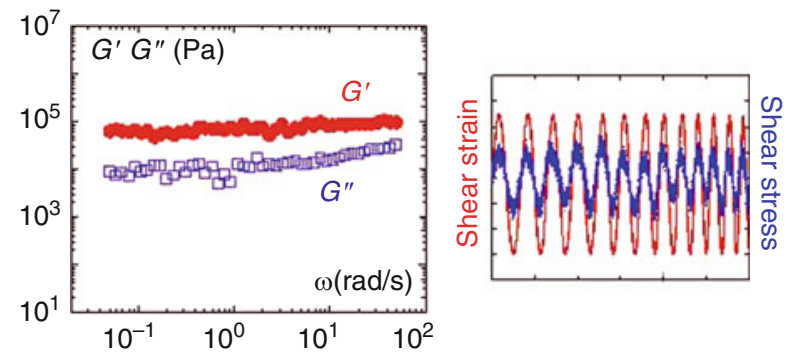

Fig. 1 The shear elastic $\left(G^{\prime}\right)$ modulus of polymer melts exhibits finite shear elastic response at low frequency at the submillimeter scale. Left figure: dynamic response of a polybutylacrylate $(\mathrm{Mw}=47,000 \mathrm{Da})$ at room temperature, i.e., at $90{ }^{\circ} \mathrm{C}$ above the glass transition $\left(T_{g}=-65^{\circ} \mathrm{C}\right)$ measured at $0.025 \mathrm{~mm}$ using wetting substrate (alumina). The right figure shows that the output stress wave (blue sin wave) is superposed to the input strain wave (red sin wave)

commercial equipment has quickly emerged as the discipline of characterization of the fluidic state and of deformable materials at a macroscopic scale (Ferry 1981). Its principle consists in the analysis of the response transmitted by the material subjected to a dynamic mechanical shear stress. The shear stress being transferred to the sample by the simple contact to the substrate the technique is entirely dependent on the energy of interaction between the material and the substrate. However, very few studies have been aimed at analyzing the influence of the impact of the interaction between the support (substrate) and the sample on the rheological behavior. Despite the early works of Young-Dupré (Young 1805), it has long been regarded that the interaction between a liquid and a surface has no effect on the measurement, as it seems obvious that a liquid wets any surface and that the obtaining of reproducible results validate these assumptions.

The emergence of new disciplines combining different techniques as microrheology (Goyon et al. 2008), microtribology, dielectric relaxation (Pronin et al. 2011), NMR (Tracht et al. 1998a, b), X-ray photon correlation spectroscopy (Chushkin et al. 2008; Conrad et al. 2015), SANS measurement (Watanabe et al. 2007; Noirez 2009b; Korolkovas et al. 2019), and particle tracking velocimetry (Noirez et al. 2009b; Mansard et al. 2014), but also critical reviews of flow of molten polymers (Hatzikiriakos 2012), highlights dynamic heterogeneities and relaxation modes in fluids much lower than the terminal defined by the viscoelastic model. The impossibility of describing the fluid as a continuum from macroscopic to the molecular level invites to revisit the assumption of molecular relaxation times as a relevant parameter to describe flow mechanisms. This entry reminds briefly the premises of the viscoelastic approach in polymer dynamics and the assumptions and the difficulties inherent in an empirical approach linked to a mechanical measurement and describes an alternative strategy taking into account microtribology, adhesion, and wetting parameters to improve the quality of the rheology measurement. These developments point out that fluids contain "static" (low-frequency) shear elasticity away from phase transition temperature and at a macroscopic length 
scale (Fig. 1). These solid-like properties usually neglected in the fluidic state open the routes to new theoretical and experimental developments based on collective dynamics. Examples of spectacular low-frequency mechano-responsive effects due to the static elasticity are shortly presented including both simple liquids and the fluidics of physiologic liquids.

\section{Empirical Origins of the Viscoelastic Approach in Polymer Dynamics and Limitations}

Polymer science and technology have made considerable increasing proportions in the twentieth century. Characterizing mechanical properties of polymers raised new challenges since the question of viscous-like or solid-like property appears to be relative to the patience of the observer. The foundations of a systematic study versus time, i.e., versus frequency, were laid. The principle of a systematic time-dependent measurement relating the strain to the stress was achieved by Weissenberg and coworkers (Weissenberg 1948) designing the future rheometer. The commercialization of instruments for rheology has accelerated the promotion of the technique that rapidly became the method to expertise the dynamic properties of viscoelastic fluids. The frequency dependence of experimental curves served as the basis for theoretical approaches for polymer physics (Doi and Edwards 1978; Ferry 1961; de Gennes 1971) on the basis of the earlier Rouse model (Rouse 1953) linking the perturbation of chain configurations upon shear stress to the viscoelastic response of noninteracting polymer chains in dilute solutions. The concept of a direct link between the rheological viscoelastic properties and the molecular structure was established (Fig. 2).

Polymer viscoelasticity theory is thus essentially based on a single-chain picture where the frequency dependence of the viscoelastic response is supposed to reproduce the intrachain configuration relaxation under shear stress, i.e., the single-chain dynamics. This fundamental assumption implies correlatively that the interchain

Fig. 2 The Rouse model proposes that the viscoelastic spectrum is produced by the distribution of configurations of noninteracting polymer chains in a dilute solution (Rouse 1953)

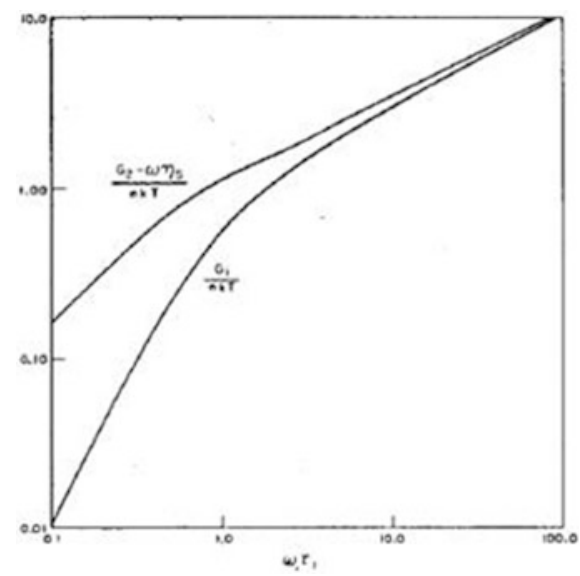


interactions are negligible. In the frame of the Rouse model, the chains are sufficiently diluted in solution to not interact from each other. The solvent contribution is treated as an independent parameter uncoupled to the chain contribution, while it is the necessary vector of transfer of the stress from and to the substrate.

The chain is seen as a succession of freely joined elastic springs. Each spring represents a group of monomers (blob) unperturbed by others (Gaussian statistics). In this frame, the generalized Maxwell model can be applied, and the viscoelastic curve is assimilated to it:

$$
\begin{gathered}
G^{\prime}(\omega)=n k T \sum_{p=1}^{Z} \frac{\omega^{2} \tau_{p}^{2}}{1+\omega^{2} \cdot \tau_{p}^{2}} \\
G^{\prime \prime}(\omega)=\omega \cdot \eta_{s}+n \cdot k T \sum_{p=1}^{Z} \frac{\omega \cdot \tau_{p}}{1+\omega^{2} \cdot \tau_{p}^{2}}
\end{gathered}
$$

where $G^{\prime}(\omega)$ and $G^{\prime \prime}(\omega)$ are the shear elastic modulus and the viscous modulus, respectively, $\eta_{s}$ is the solvent viscosity, $\tau_{p}$ is the relaxation time of the group $p, Z$ is the number of springs, $n$ is the number of chain per volume unit, and $T$ is the temperature. At low frequency, $G^{\prime}$ scales as $\omega^{2}$ with a zero-frequency limit corresponding to the absence of shear elastic component. The zero-frequency limit of the viscous part gives also rise to a collapse $\left(G^{\prime \prime} \cong 0\right)$. The viscoelastic model for fluids predicts neither shear elasticity nor viscous modulus at the zero-frequency limit (Fig. 3).The Rouse model for diluted solutions is no more valid at high frequencies because the $\omega$-scaling of the solvent contribution of the viscous term is limitless that challenges the solid-like response function that every fluid does reach at sufficiently high frequencies (Scarponi et al. 2004; Hansen et al. 2013; Hasegawa et al. 2016). Therefore the Rouse model can only account for a low-frequency branch of the response.

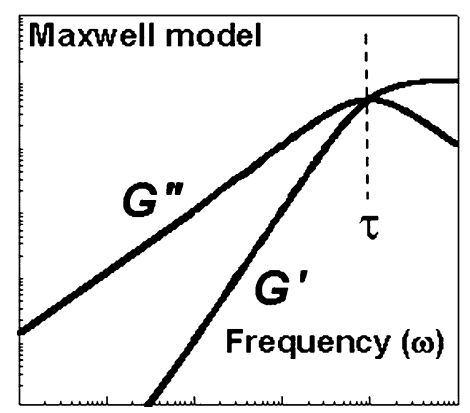

Fig. 3 Polymer melts, concentrated to diluted polymer solutions (conventional measurements), exhibit similar dynamic relaxation spectra which are supposed to be linked to different chain configurations upon applying shear stress (single-chain dynamics model). The low-frequency behavior is characterized by $G^{\prime \prime}(\omega)$ and $G^{\prime}(\omega)$ fitting with $\omega$ and $\omega^{2}$ scaling, respectively (Maxwell model) 
The distinction between a viscoelastic liquid and viscoelastic solid is introduced by adding a finite term $G_{e}$, independent of the frequency, to the shear modulus so that it does not vanish at low frequency but keeps an invariant shear elastic value (Ferry 1981):

$$
G^{\prime}(\omega)=n k T \sum_{p=1}^{Z} \frac{\omega^{2} \tau_{p}^{2}}{1+\omega^{2} \cdot \tau_{p}^{2}}+G_{e}(T)
$$

$G_{e}$ expresses infinite relaxation times.

From an experimental point of view, the distinction between viscoelastic fluids and solids remains relative since the measuring devices benefit from constant instrumental improvements both in terms of frequency range and of sensitivity. Weak shear elastic moduli that could not be hitherto detected might be now detected and identified. Moreover, it has been demonstrated that the noise inherent to this type of measurement is a source of failure that does not enable to guaranty the uniqueness of the solutions of the generalized Maxwell model (Jalocha et al. 2015).

Finally, while the representation of the mechanical behavior of (viscoelastic) fluids using the generalized Maxwell model is simple, the vanishing of $G^{\prime}$ and $G^{\prime \prime}$ at zerofrequency limit is debated. It challenges the liquid dynamic description that takes into account the weak force of intermolecular interactions that determine the cohesion energy (Lugorski Karle et al. 1944). The cohesive state of the liquid results from the interacting nature of its constituting molecules. Van der Waals, hydrogen-bonding, and polar interactions govern the forces of the boundary interactions. Each particle is submitted to its activation energy. The particle motion becomes allowed when the friction forces transfer an impulsion that overcomes the mobility energy of the molecules. In other words, the fluid is supposed to resist to the flow, before flowing. The energy threshold is nonzero (Ediger et al. 1996) and hardly compatible with a description in terms of an absence of both viscous and elastic quiescent properties when the frequency lowers to zero. We present here some experiments that point out the existence of nonzero shear elasticity at low frequency at the submillimeter thickness. This "static" elasticity does not preclude conventional viscoelasticity but relocates it in a wider spectrum depending on the sample dimensions.

\section{Hidden Experimental Difficulties Inherent to a Viscoelastic Measurement and to the Determination of a Viscoelastic Time}

Rheological measurements are generally carried out applying a small amplitude oscillatory motion to keep the sample as close as possible to equilibrium conditions (in agreement with the causality-linearity principles). The fluid is placed in contact with and between two surfaces (generally disk-like fixtures in rotation symmetry), one oscillating, the other one fixed measuring the shear stress transmitted by the sample via a sensor (Fig. 4). From the difference between the input and the output signals, two parameters are extracted in terms of frequency-dependent shear moduli 
a

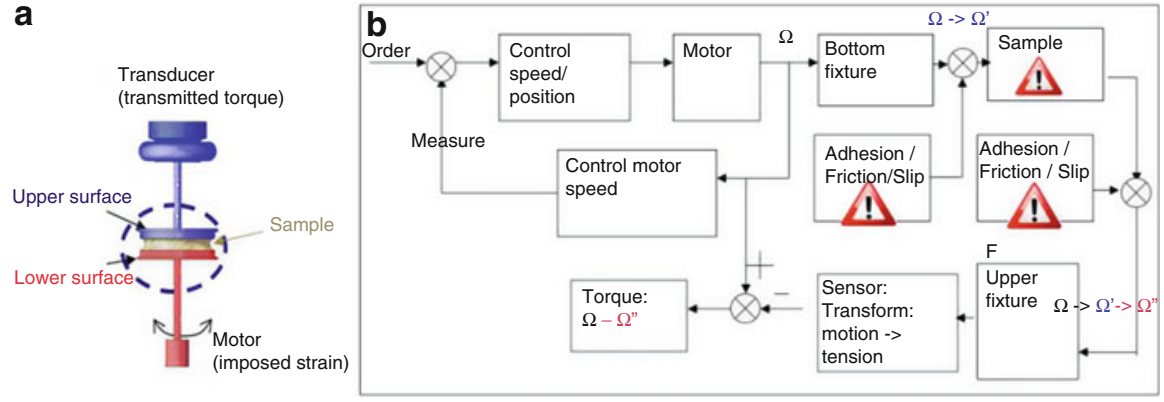

Fig. 4 (a) Scheme of the experimental setup. The sample is placed between two surfaces. The shear strain is transmitted to the sample via molecular contacts with the lower surface which is animated by an oscillatory motion of given frequency $\omega$ and amplitude $\gamma_{0}$. The shear stress is communicated along the sample thickness and is transferred via molecular contacts to the second surface coupled to a force (here a torque) sensor (real imposed strain geometry). (b) Simplified scheme of the transmission chain of the information in dynamic relaxation (in imposed strain geometry): the transmission of the shear strain to the sample and the transmission of the shear stress strain of the sample to the sensor are entirely tributary of the interaction forces between the liquid and the surface onto which it is deposited. This transmission chain can be formalized as follows:

$\Omega$ is the imposed shear torque.

$\Omega^{\prime}$ is the shear torque transmitted to the sample.

$\Omega^{\prime}=\Omega-$ losses from the surface to the sample (slip).

$\Omega^{\prime}$ is the shear torque received at the sensor.

$\Omega^{\prime \prime}=\mathrm{F}\left(\Omega^{\prime}\right)$ - losses of the sample at the surface where $\mathrm{F}$ is the transfer function by the sample.

where the shear elastic modulus $G^{\prime}(\omega)$ and the viscous modulus $G^{\prime \prime}(\omega)$ are the inphase and out of phase components, respectively.

This stress response is interpreted as the Fourier transform of the stress relaxation function $G(t)$ of the quiescent state: $G^{\prime}(\omega)=\omega \int_{0}^{\infty} G(t) \sin \omega t$.dt, $G^{\prime \prime}(\omega)=\omega \int_{0}^{\infty} G(t)$ $\cos \omega t$.dt. Under linear strain-stress conditions, the shear stress response does reproduce the applied strain wave; i.e. it is a simple harmonic function of the applied wave. $G^{\prime}>G^{\prime \prime}$ indicates a solid-like behavior, while $G^{\prime \prime}>G^{\prime}$ indicates a viscous or flow behavior. Liquids and viscoelastic liquids are characterized by a flow behavior at low frequency, typically within $0.1-10^{2} \mathrm{~Hz}$.

The surface interactions have a key role for the validity of the stress measurement.

In a conventional measurement (millimeter thickness sample probed using aluminum, stainless steel, or glass substrates), the dynamic relaxation spectrum of polymers in the molten state exhibits, versus frequency, the typical Maxwell viscoelastic response (Ferry 1961). At low frequencies, the response is depicted by a $\omega$-scaling decrease of the viscous modulus $\left(\mathrm{G}^{\prime \prime}\right)$ and a $\omega^{2}$-scaling of the elastic modulus $\left(\mathrm{G}^{\prime}\right) . \mathrm{G}^{\prime}$ being negligible compared to the viscous modulus (Fig. 5a bottom), the flow behavior thus describes the frequency part where the viscous component becomes important. The interception of the two curves defines the terminal time $\tau_{t}$, i.e., the largest time before the material enters in a flow regime. This characteristic time is interpreted as the longest molecular relaxation time (Rouse model). 

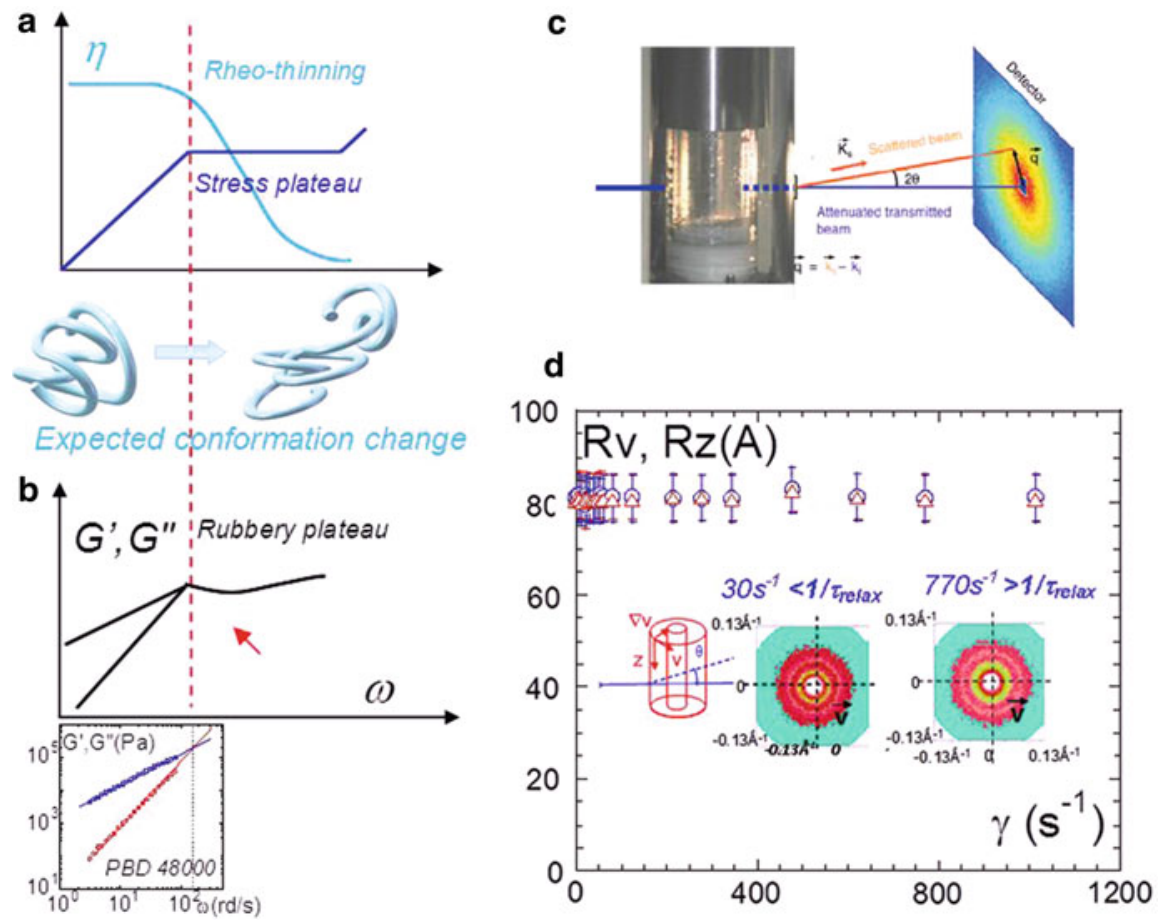

Fig. 5 (a) The upper figure schemes the viscosity $\eta$ and the shear stress $\sigma$ versus shear rate (flow curve). The rheofluidification zone (plateau) is interpreted by a shear-induced alignment of the chains. The bottom scheme shows the correspondence between the viscoelastic behavior and the flow curve with the interception of $\omega$ and $\omega^{2}$ scales of the viscoelastic curve defining the viscoelastic terminal time. (b) Conventional dynamic relaxation measurement of the macroscopic terminal behavior of the polybutadiene (PBD1,4). The reptation time is $\tau_{\text {relax }}=0.7 \times 10^{-2} \mathrm{~s}$ at $26{ }^{\circ} \mathrm{C}$ (cone-plate aluminum fixtures ARES rheometer). (c) Photograph of the PBD melt filling the Couette cylinder (gap thickness: $0.1 \mathrm{~mm}$ ) and indicating that it is strongly shear stressed at $500 \mathrm{~s}^{-1}$ (shear-thinning regime). (d) Evolution of the radius of gyration of the PBD1,4 along the velocity (Rv) and the neutral axis (Rz) versus shear rate. The insets show the $2 \mathrm{D}$ neutron scattering patterns recorded at $30 \mathrm{~s}^{-1}$ and $770 \mathrm{~s}^{-1}$ (respectively, below and above the conventional terminal relaxation time) showing unchanged isotropic form factor of the polymer chain (Reprinted with permission from Noirez et al. 2009a)

However, the apparent generic adequacy of the viscoelastic measurements to the Maxwell model should not hide the experimental divergences about the terminal time $\tau_{t}$. Over large time scale relaxations (Boué et al. 1987; Wang 2006), unexplainable spectacular flow instabilities (spurt effect, "shark-skin" instability, shear-induced transitions) (Graham 1999; Pujolle-Robic and Noirez 2001), heterogeneous flows, and slippage at the boundaries (Mansard et al. 2014; Metivier et al. 2012) continue to feed a debate about the pertinence of the viscoelastic relaxation times to describe molecular dynamics over decades (Rault 1987; Litinov et al. 2013). The absence of chain deformation under steady-state shear flow in polymer melts at shear rates exceeding the inverse of the viscoelastic time (Watanabe et al. 2007; Noirez et al. 2009b - the photograph of Fig. 5 snapshotted at shear rates much larger 
than the inverse of the relaxation time shows optically a shear-thinning effect while there is no chain deformation) questions the entanglement/disentanglement concept.

These observations point out the shortcomings of the macroscopic description in terms of molecular relaxation times while other works highlight nonlinear effects or highlight collective behavior and multiple intermolecular interactions.

The recent consideration of the boundary conditions between surface and fluid interactions in rheology measurements has proven that the viscoelastic response is not universal but strongly influenced by the wetting or the anchoring conditions (Mendil 2006; Noirez, Baroni 2010). A totally different response, stronger and exhibiting a solid-like response, is obtained using total wetting conditions. The boundary conditions thus govern the efficiency of the transmission of the stress to the sample and play a major role in the quality of the measurements. It will be demonstrated that the optimization of the interaction between the surface and the material extends the dynamic relaxation spectrum and that the Maxwell viscoelastic response is actually only a part of a wider dynamic response.

\section{Optimizing the Stress Transmission in Viscoelastic Measurements and Scanning the Submillimeter Scale Response}

Up-to-date progresses in rheology instrumentation allow the access to the measurement of the shear modulus with a high precision over six decades of magnitude. These improvements have considerably widened the frequency window and the access to very low stress moduli. Therefore, the detection of properties that would not been considered at the time of the first concepts of the viscoelasticity become accessible.

These technical improvements can be conjugated to an optimization of the stress transmission by working on interfacial fluid/solid boundary conditions. The surface parameter is rarely taken into account. However, the validity of a dynamic relaxation experiment is entirely depending on the efficiency of the stress transmission which is ensured by the molecular contact forces between the sample and the surfaces. Because of symmetry reasons, interfacial energy differs from the 3D properties. The ideal boundary conditions correspond to lower the energy gap between two different media; i.e., the surface energy has to be as high as possible. A good criterion is the observation of a total wetting of the fluid onto the substrate (de Gennes et al. 2005 - Fig. 6b). The total wetting maximizes the molecular interactions to the surface. By filling voids, roughness, and asperities, it prevents the slippage.

The discipline of rheology has grown ignoring the fluid/substrate boundary conditions on the dynamic measurement, treating in an equal way, wetting or non-wetting, hydrophilic or hydrophobic surfaces. Rheology supposes that the fluid wets adequately the metallic surfaces of the fixtures (generally made of aluminum or stainless steel). However, the determination of the contact angle shows that metallic substrates do not guaranty an optimal wetting (Fig. 6b, c). As a consequence of partial wetting conditions, the interfacial forces are reduced to 
a Medium surface energy: metal, glasses, PVC

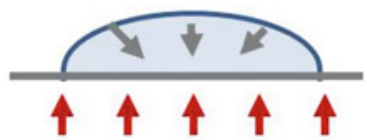

\section{Partial wetting}

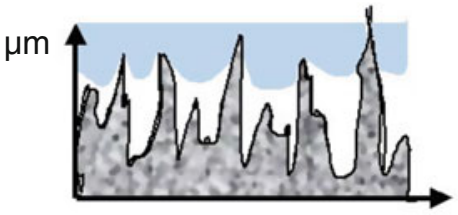

b Very high surface energy: Functionalized surfaces, some metal oxides

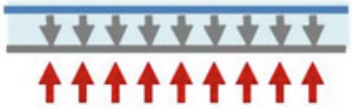

Total wetting

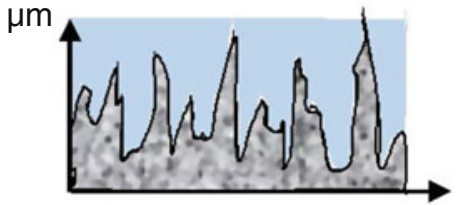

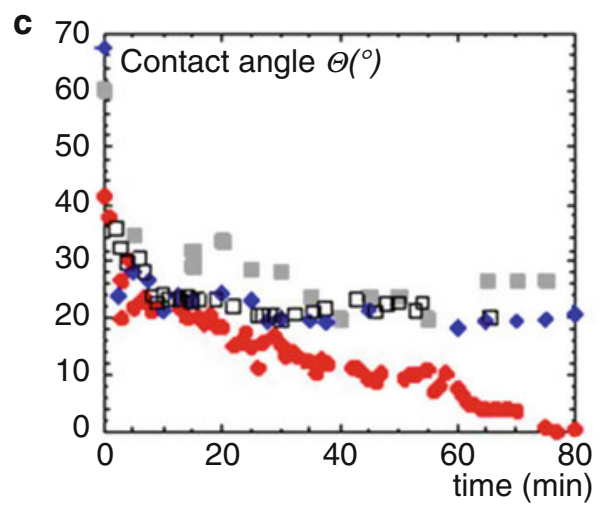

Fig. 6 The wide majority of substrates (aluminum, stainless steel, glasses) exhibits partial wetting conditions. Only few fluid/substrate pairs fulfill the total wetting conditions. These conditions depend on the nature of both the fluid and the substrate. (a) The partial wetting is characterized by a finite Young contact angle and an incomplete wetting of the surface asperities (bottom profilometry scheme). (b) The zero-contact (macroscopic) angle of the total wetting ensures a complete wetting of the surface asperities (bottom profilometry scheme). (c) The contact angle is the angle where a liquid/air interface meets a solid surface. It quantifies the wettability of a solid surface by a liquid and the slippage tendency using the Tolstoi's relationship $b \sim \exp \left(\frac{\sigma^{2} \gamma(1-\cos \theta)}{\mathrm{kT}}\right)-1$ where $b$ is the slip length, $\sigma$ a molecular constant, and $\gamma$ the surface tension (Tolstoi 1952). The evolution of the contact angle versus time shows that the alumina substrate (red points: $\bullet$ ) provides a total wetting $(\theta=0)$, while the angle reaches a stationary value of $20^{\circ}$ (partial wetting) for aluminum $(\diamond)$, glass $(\square)$, and stainless steel $(\square)$.

molecules remaining in contact with the substrate, and the stress is not fully transmitted to the sample.

Using the total wetting boundary conditions (alumina surfaces) and a conventional rheometer (Mendil et al. 2006; Baroni et al. 2005, 2010; Wang et al. 2007), a low-frequency solid-like response emerges at the submillimeter scale in a series of polymer melts (Fig. 7). This observation was carried out on several ordinary 

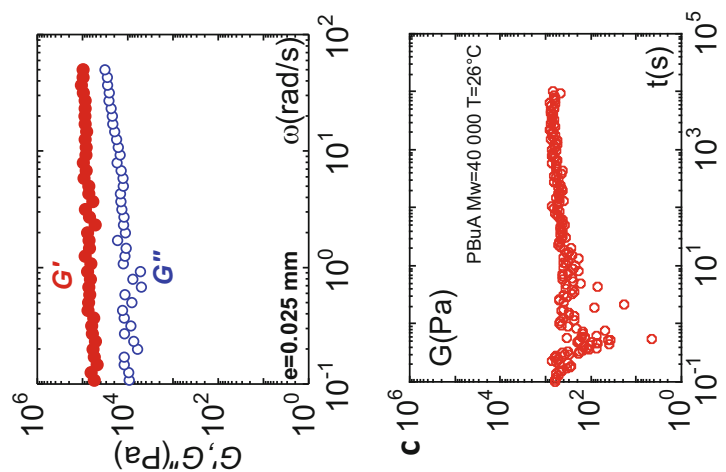

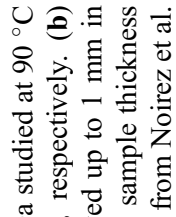
คิ

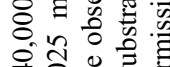
คำ จ

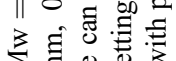

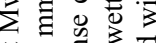

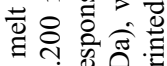

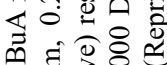

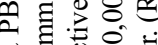
๘ 0 웜 옹유월 过

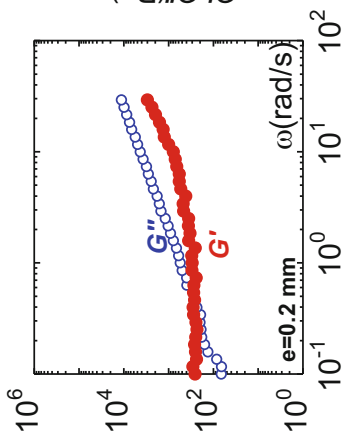

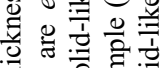
요용

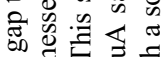

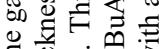
‡. on \pm 응 망 을

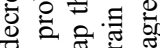

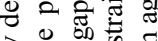
तิ 仓ें

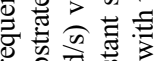
כ"כ)
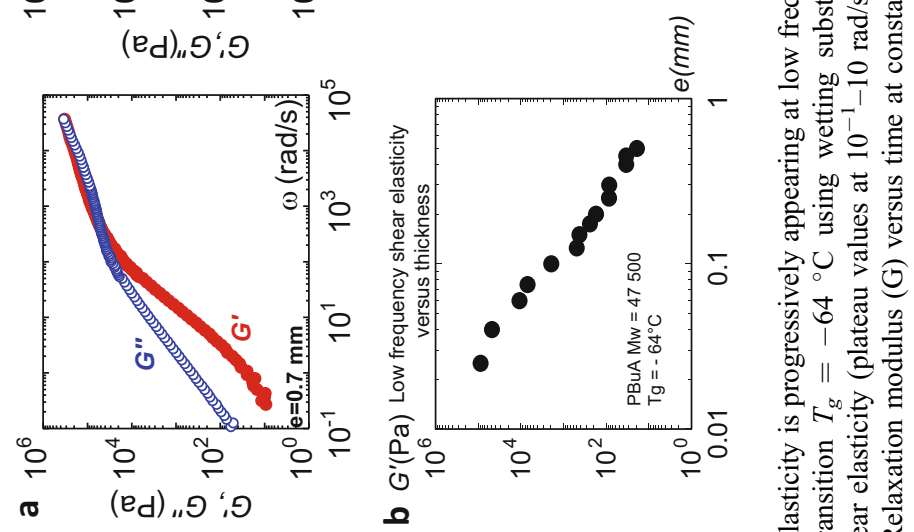

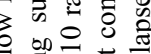
洰西 일을용 क्षै

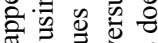

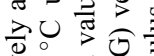

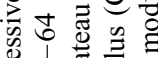

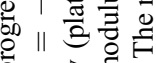

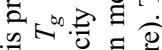

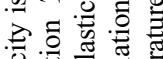
矛芯 卷出的导

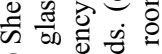
సิ

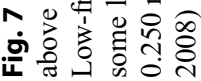


polymers (polyacrylates, polymethacrylates, polybutadienes, etc.) tested from smaller to higher molecular weights (unentangled and entangled) up to high macroscopic scales (up to $0.5 \mathrm{~mm}$ gap thickness) and at about $100{ }^{\circ} \mathrm{C}$ away from the glass transition and is in agreement with piezorheometer measurements carried at lower thicknesses (Derjaguin 1983; Derjaguin et al. 1989; Badmaev et al. 1983; Collin 2002).

\section{From Surface-Induced Solidification to the Identification of the Submillimeter Shear Elasticity}

Low-frequency elastic behaviors have been reported at several occasions. Since 1991, Granick and coworkers (Hu et al. 1991; Demirel and Granick 2001; Zhu and Granick 2004) measured, at the molecular scale or a multiple of that, a solid-like component at nanoscales by SFA (Surface Force Apparatus) using mica surfaces. Close to a surface, these results were generally interpreted as resulting from surface-induced effects. The disappearance of the solid-like response at larger thicknesses is usually interpreted by a transition from surface to bulk properties. However the Mica (muscovite) surface does not provide strong liquid-substrate interactions (partial wetting) with organic fluids. Such boundary conditions are similar to those for conventional viscoelastic measurements. The very first analysis in terms of physical property is due to Derjaguin (Badmaev et al. 1983; Derjaguin et al. 1989) revealing in different fluids including polymers and liquid water, an elastic response at the micron scale that is interpreted as an intrinsic property generated by intermolecular interactions. At a larger scale, Collin et al. reported, using treated glass surfaces and small strains delivered by a piezorheometer, on a gel-like response up to $50 \mu \mathrm{m}$ thicknesses in low molecular weight polystyrene melts that was interpreted as a reminiscence of the glass transition, i.e., clusters of finite size (Collin and Martinoty 2002). Since 2006 the use of optimized fluid/ substrate wetting conditions has facilitated the measurement of the low shear elasticity, generalizing its identification to ordinary liquids and confirming the probable origin due to intermolecular forces. The wetting protocol opens an easier access to the solid-like response usually hidden in conventional measurements of viscoelastic fluids.

Figure 8 illustrates the response obtained using full wetting conditions for an H-bond oligomer poly(propylene glycol) (PPG4000) at room temperature. The flow behavior obtained conventionally on metallic substrate is here replaced by an elastic response $\left(G^{\prime}\right.$ and $G^{\prime \prime}$ nearly independent of the frequency and with $\left.G^{\prime}>>G^{\prime \prime}\right)$. The signal analysis indicates that the shear stress wave is superposed to the strain wave, confirming the instant transmission of the stress characterizing a solid-like behavior. This result is coherent with the conclusions in terms of elastic contribution carried out on the same liquid on the basis of the dynamic of the capillary waves (Chushkin et al. 2008). 

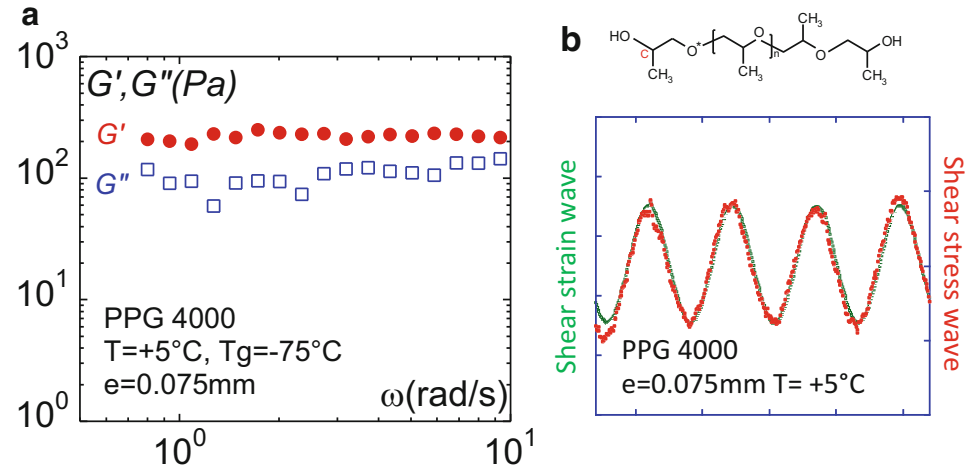

Fig. 8 (a) Frequency dependence of the elastic $G^{\prime}(\omega)$ :• and viscous moduli $G^{\prime \prime}(\omega)$ : $\square$, measured for a glass former liquid (poly(propylene glycol) - PPG4000, $M W=4000 \mathrm{Da}, T_{g}=-75^{\circ} \mathrm{C}$ ) at $\mathrm{T}=+5{ }^{\circ} \mathrm{C}(0.075 \mathrm{~mm}$ gap thickness - alumina plate-plate fixtures). (b) Superposition of the strain (green points) and the stress (red points) waves highlighting the instant response of the liquid

\section{Generalization of the Submillimeter Shear Elasticity to Fluids and Liquids}

The low-frequency shear elastic regime is observed away from the glass transition (above a hundred degrees), on typically $0.025-0.500 \mathrm{~mm}$ sample thicknesses, thus in a liquid state where $G_{e}$ is supposed to be zero. Using improved liquid/ substrate boundary conditions (i.e. high energy surfaces like the alumina to provide total wetting), the observation of a terminal elastic response has been also reported in glass formers ((PPG-4000; see Fig. 8), o-terphenyl (Noirez et al. 2011), glycerol (Noirez and Baroni 2010), and ordinary alkanes (Noirez et al. 2012)) and even on liquid water. The low frequency shear elasticity cannot be interpreted by entanglement effects and the vicinity of the glass or of a crystalline transition but is due to intermolecular interactions ensuring the liquid cohesion. Being measured at several tenths or hundredths of millimeter and reaching several thousands Pascals (polymer melts), the low-frequency shear elasticity cannot be interpreted by a surface-induced solidification. A solid-like response is even reported up to the millimeter length scale in the isotropic phase of a liquidcrystalline polymer (Noirez 2005), while the molecular dimensions are less than $100 \AA$. The strong anchoring of the liquid crystal molecules reinforces the boundary contacts between the fluid and the substrate whereby the stress is transmitted and facilitates the measurement (Fig. 9). Gallani et al. observed, using the molecular displacement strain produced by the piezorheometer, an "abnormal" viscoelastic behavior on tens micron sample thicknesses in the isotropic phase of a liquid-crystalline polymer substrates using a surface treatment (Gallani et al. 1994; Martinoty et al. 1999). 
a

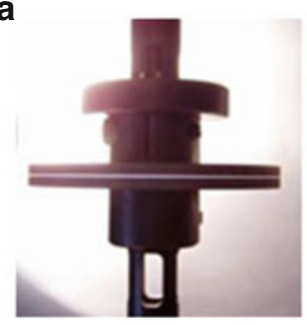

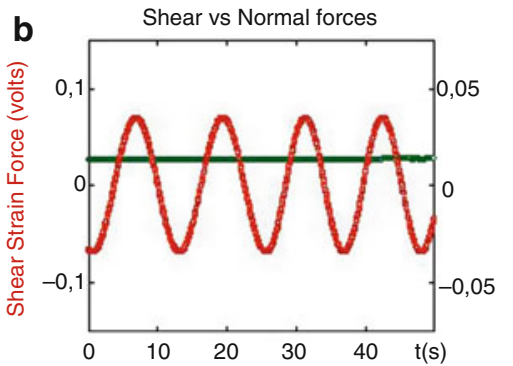

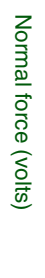

\section{C}
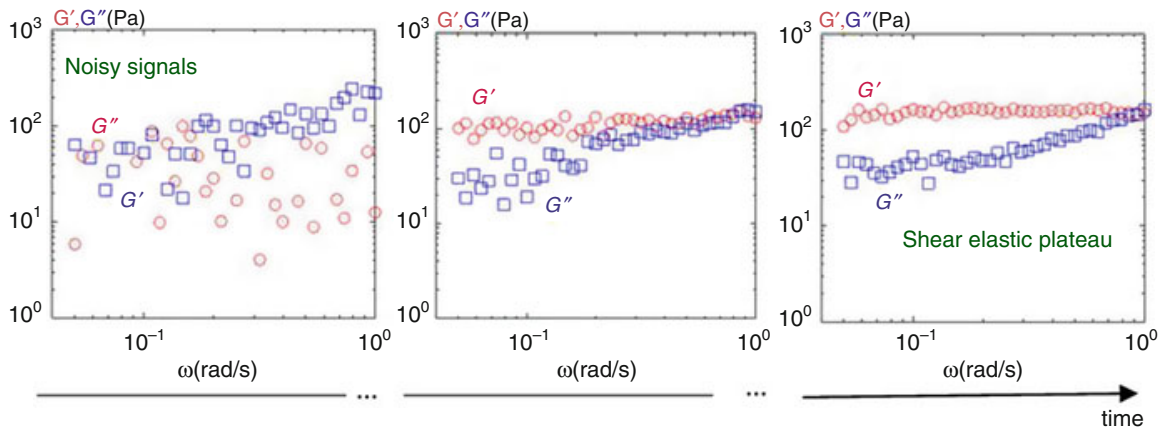

Fig. 9 (a) Photograph of the alumina fixtures (surface roughness $<1 \mu \mathrm{m}$, parallelism $<10^{-3} \mathrm{rad}$ (zero-contact method via normal force and no-light transmission), diameter $40 \mathrm{~mm}$ ). (b) Shear strain force (red data points) and normal forces (green data points) are simultaneously measured during the oscillatory shear strain motion using two multimeters connected to the shear strain and the normal force transducers (ARES device, $\omega=0.1 \mathrm{~Hz}, \gamma=5 \%, e=0.1 \mathrm{~mm}$, liquid water at room temperature). (c) Evolution of the viscoelastic signal with time: the liquid interaction with surface is progressive (migration of bubbles, interaction with surface). Data points recorded on a polymer melt at sub-millimeter thickness using alumina surface (total wetting conditions) - P. Kahl, PhD thesis (https://www.theses.fr/2016LEMA1002.pdf).

\section{Shear Elasticity and Surface Tension}

The access to low-frequency shear elasticity is facilitated by minimizing any process that alters the stress transmission. Degassed fluids and wetting protocol enable a better stress transfer from the surface to the sample compared to conventional measurements. It is however important to point out that the increased moduli values are not due to interfacial surface tension (Johnston et al. 2013). The surface tension (peripheral (air/fluid) or surface (fluid/substrate)) is supposed to not change during the oscillatory motion. These conditions are fulfilled when the sample volume is kept constant during the shear measurement [complete and homogeneous (without bubbles) filling of the gap, rigorous parallelism of the surfaces, and close equilibrium conditions (low strain amplitudes)]. The simultaneous measurement of the normal 
force is a direct access to the volume variation (or displacement) during the oscillatory stress measurement and therefore is directly related to surface tension. Figure 9 illustrates the values of the normal force and of the shear stress measured simultaneously and displayed on the same scale. No variation of the normal force is observed during the measurement, while the sinusoidal waves corresponding to the shear stress or the shear strain are easily identifiable.

The (linear) low-frequency shear elasticity is accessible upon low displacement excitation (low strain amplitude regime or low sample thickness) and is prior to the conventional viscoelastic regime which is obtained at larger strain amplitudes (next paragraph). A variable waiting time is required prior the terminal elastic behavior emerges. Close equilibrium conditions are thus much more fulfilled for the low-frequency elasticity measurement than for the conventional viscoelastic regime obtained at larger strain amplitudes and even more than in the so-called LAOS (large amplitude oscillatory strain) regime where interfacial surface tension parameters might be integrated.

\section{Relationships Between Low-Frequency Shear Elasticity and Conventional Viscoelasticity}

If the shear elasticity of the fluid is observable at submillimeter scale by improving the wettability of the substrate, it is progressively lost by increasing the strain amplitude (or by increasing the sample thickness) giving rise in the case of polymer melts to the conventional flow behavior identical to partial wetting conditions (Fig. 10). A transition from total to partial wetting conditions is thus achieved by increasing the strain amplitude. The strain-induced transition can be interpreted as a selection process with respect to the boundary contacts between the polymer and the substrate. Fast relaxation time contacts are easily restored and give rise to the conventional flow behavior, whereas long relaxation (solid-like) contacts bear only small strains and exhibit the elastic response. The elastic response corresponds to a primary linear regime. The viscoelastic behavior is thus the nonlinear product of the first elastic regime.

Similar shear elasticity collapses are observed in other viscoelastic fluids or liquids as, for example, in the case of the heptadecane (Fig. 11).

The identification of a finite shear elasticity indicates a collective (intermolecular) response. It rules out an interpretation in terms of single molecular response solely due to intrachain elasticity modelled by the Maxwell function (viscoelasticity theory). Low-frequency shear elasticity is the firm demonstration that intermolecular interactions contribute also to the dynamic response. This scheme is in agreement with the identification of finite shear elasticity at low frequency on low molecular liquids as illustrated here in the case of the heptadecane (Fig. 12). 


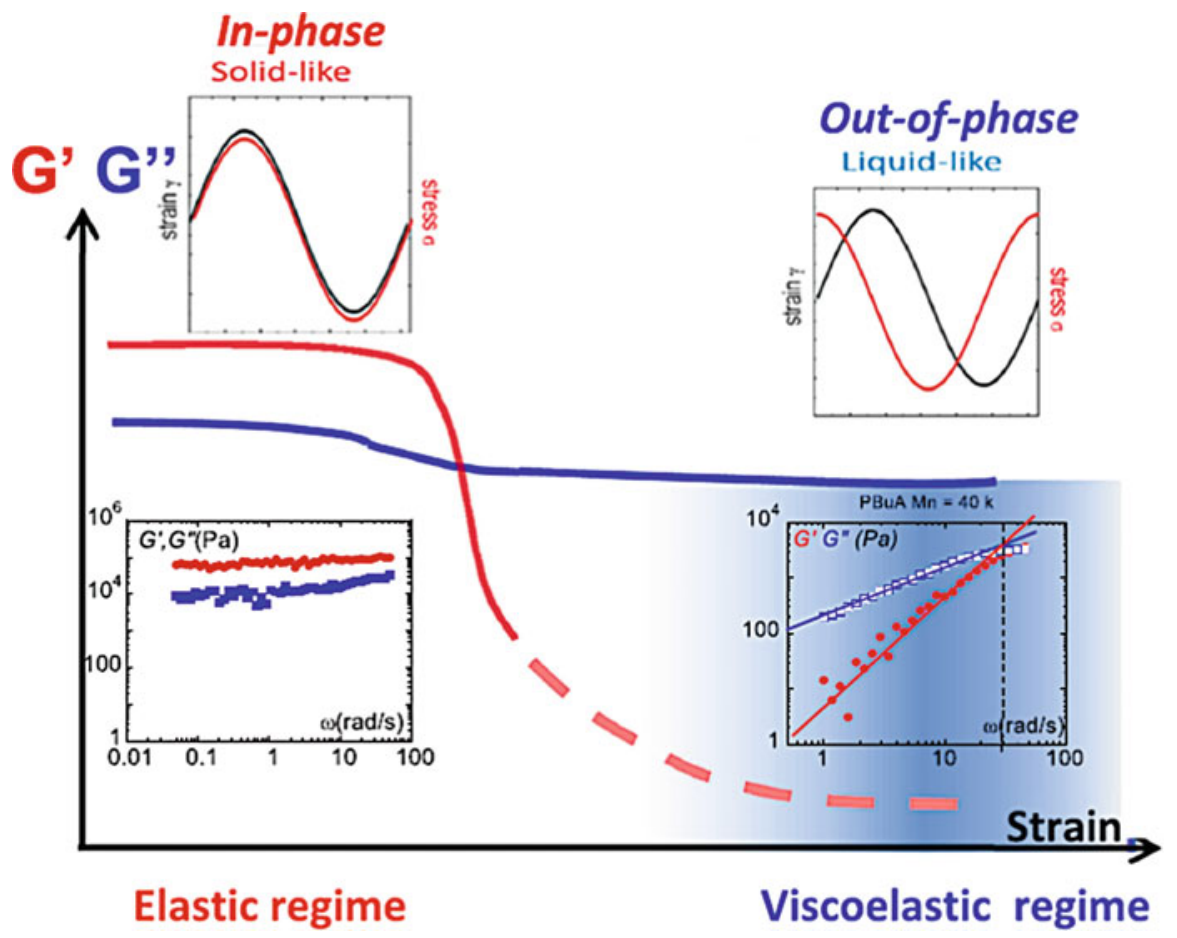

Fig. 10 Scheme gathering the evolution of the shear elasticity $\left(G^{\prime}\right)$ versus strain amplitude (the inserts display the dynamic spectra of the polybutylacrylate PBuA $M w=47,000 \mathrm{Da}, T=25^{\circ} \mathrm{C}, 0.060 \mathrm{~mm}$ gap thickness, plate-plate geometry, alumina fixtures) $\gamma:(\bullet) 0,5 \%,(\triangleright) 1 \%,(\nabla) 2 \%,(\triangle) 3 \%,(\triangle) 5 \%$, $(\diamond) 10 \%,(\square) 20 \%,(\triangle) 30 \%,(\diamond) 50 \%,(\nabla) 150 \%$. The dotted line shows the $\omega^{2}$ scale

In this context and considering the major role of the intermolecular interactions in liquids (Hansen and McDonald 1991; Chandler and Andersen 1972), it is particularly relevant to consider dynamic approaches taking into account also interchain non-covalent interactions and not only intrachain interactions. Very few studies examine the possible contribution of intermolecular chain interactions in polymer physics. Despite the continuum condition from the unentangled state to the molecular fluid, the single (noninteracting) chain concept is generally kept to describe the low molecular weight polymer dynamics (Rouse model). However, Weiner and Fixman (Gao and Weiner 1991; Fixman 1991) demonstrate by computer simulation that the dominant contribution to the viscoelasticity would be due to excluded volume interactions, i.e., interchain forces. Their simulation results are in agreement with NMR experiments (Deloche 1986; Sotta et al. 1987) showing that a free chain is influenced by an anisotropic chain medium. Other recent computer simulations still evidence the relevance of considering intermolecular interactions in unentangled polymer fluids (Guenza 2002a, b). To our knowledge, the early prediction of long-range elastic correlations in the (generic) liquid state is 


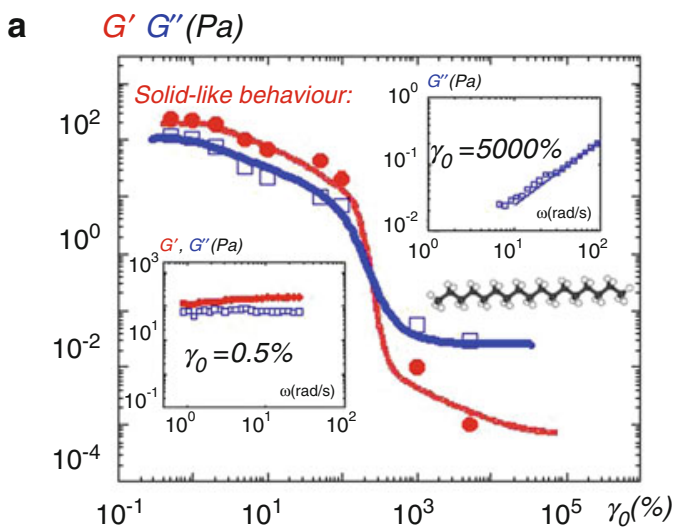

b

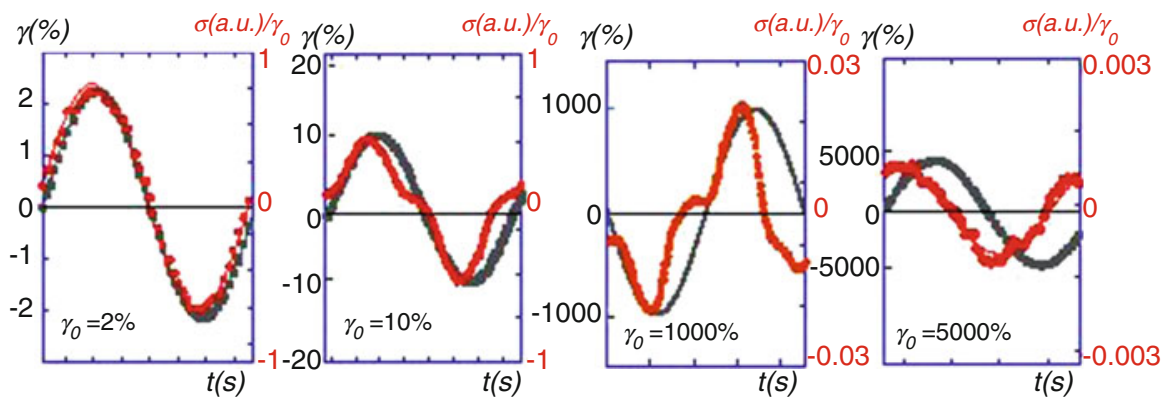

Fig. 11 Shear strain dependence of liquid $\mathrm{n}$-heptadecane at $0.058 \mathrm{~mm}$ gap thickness including the low frequency shear elasticity at low strain (a) Transition from solid-like to viscous behavior induced by increasing the strain amplitude $\left(\gamma_{0}\right)$. The data points display the shear $\left(\mathrm{G}^{\prime}(\bullet)\right)$ and the viscous $\left(\mathrm{G}^{\prime \prime}\right.$ ( $\square$ )) moduli at $1 \mathrm{rad} / \mathrm{s}$ frequency. At low strain amplitudes, a solid-like behavior is observed. The moduli in the intermediate nonlinear regime are not presented since the signal is not a sinusoidal wave. At very large strain amplitudes, an apparent viscous behavior is displayed. This right part of the spectrum (at right from the red right line) corresponds to the conventional viscous behavior (b) From left to right: input sin wave (: strain amplitude $\gamma(\%)$ ) and output shear stress wave (॰: shear stress: $\sigma\left(\right.$ a.u.)) corresponding to the liquid response of the n-heptadecane at $24{ }^{\circ} \mathrm{C}$ measured at different imposed strain amplitudes $\left(\gamma_{0}=2,10,1000\right.$, and $\left.5000 \%, e=0.058 \mathrm{~mm}\right)$. Similar behaviors are observed with other fluids (polypropylenglycol, water, ionic liquids, etc.). The continuous red line in the first and the last figures corresponds to a sinusoidal fit. (Reproduced with permission from Noirez et al. 2012)

due to Volino (1996). Remarkably, the shear elasticity is predicted to be dimensiondependent, which is in agreement with the observations (Derjaguin 1989; Collin 2002; Mendil et al. 2006).

The solid-like property is usually observable at low thickness and is thus hidden in conventional mechanical measurements since the conventional measurements are carried out at thicknesses of $1 \mathrm{~mm}$ or higher (which corrresponds to the asymptotic branch of the shear elasticity). The sub-millimeter shear elasticity is 
a

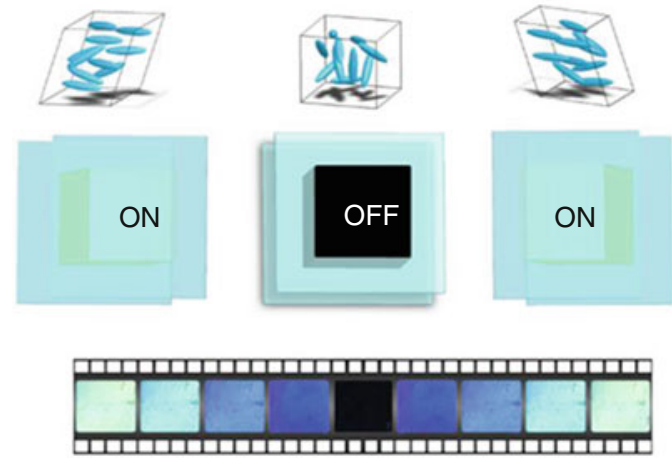

b

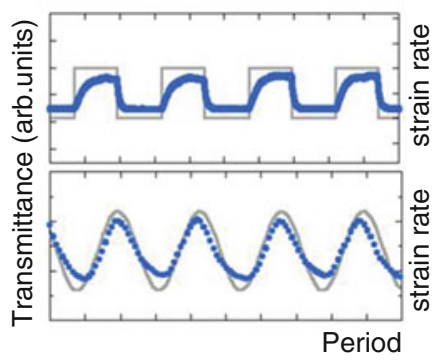

Fig. 12 (a) Snapshots of the isotropic phase of a liquid crystal polymer under low-frequency shear strain (similar effects are observed for low molecular weight liquid crystals). The different colors are photographs of the birefringence appearing during the oscillatory motion. (b) Playing with the strain wave function modulates the transmittance signal (Kahl et al. 2015 and $\mathrm{PhD}$ thesis 2016). Link for the video: https://www.youtube.com/watch? $\mathrm{v}=0 \mathrm{NPjNG6FkTE}$

however not negligible, reaching several thousands Pascals in polymer melts at low thicknesses. The consideration of the low-frequency shear elasticity opens the route to the identification of new behaviors like the mechano-responsive optical signal in the isotropic phase of liquid crystal polymers (Kahl et al. 2013, 2015) (Fig. 12), coexisting thermal shear bands and, in particular, the occurrence of shear-induced cooling in polymer melts under flow and upon applying an oscillatory shear strain (Fig. 13) (Baroni et al. 2013; Noirez et al, 2018). Coexisting thermal shear bands show that the mechanical energy do not dissipate in the thermal fluctuations and that the thermodybnamic state can be easily modified upon applying a low shear rate or a low frequency shear stress. It also demonstrate that the thermal fluctuations are not simply linked to density fluctuations but also strongly correlated to the shear elasticity and thus to the ability of changing the liquid compressibility. The shear-induced cooling has been also observed up the millimeter scale in liquid water (Fig. 13, Baroni 2013). This is very likely a generic effect that concerns particularly microfluidic conditions. Biomedicine via the transport of physiologic liquids is directly concerned. First thermal analysis on physiologic fluids are very promising proving the coexistence of cold and hot shear bands in human blood plasma submitted to an oscillatory shear flow mimicking the human heart rate (Fig. 14, Noirez, Windberger in preparation). These examples illustrate macroscopic effects induced by the long range intermolecular elastic correlations and point out the urgent need to propose a new approach integrating external parameters as the surface boundary conditions and internal parameters as local compressibility. 
a

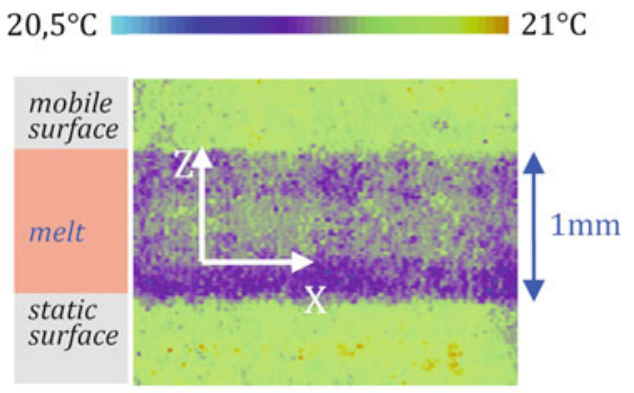

b

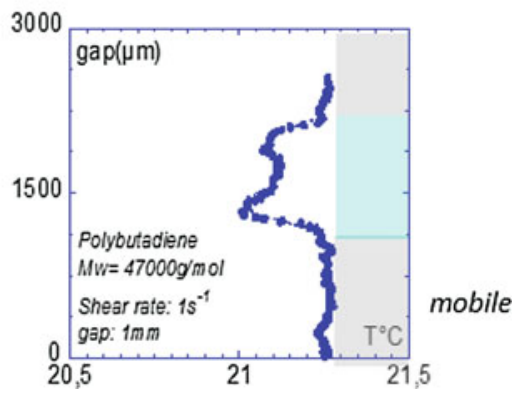

Fig. 13 (a) 2D-thermal imaging recorded in a $2 \mathrm{~mm}$ gap produced at a low shear rate $\left(1 \mathrm{~s}^{-1}\right)$ by a low molecular weight polybutadiene contrasts with the expected Newtonian flow and indicates a nonlinear behavior invisible via other techniques. (Reproduced with permission from Baroni et al. 2013). (b) Vertical section along the velocity gradient showing the temperature profile (shear induced cooling) at alow shear rate (1s-1).

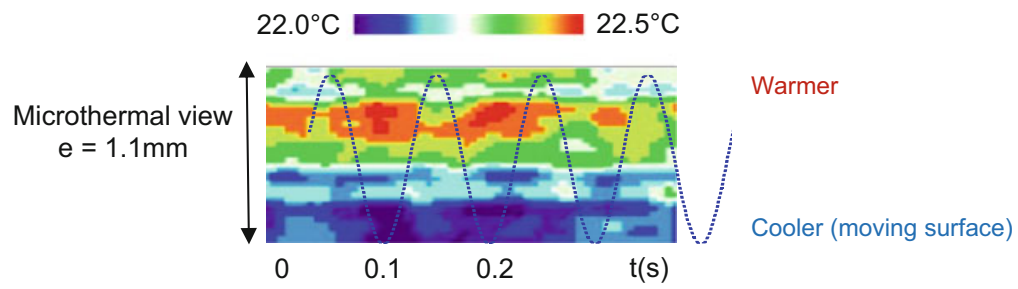

Fig. 14 Microthermal cartography of human blood plasma submitted to an oscillatory shear strain of $10 \mathrm{rad} / \mathrm{s}$ which corresponds to the heart rate (gap view, $1.1 \mathrm{~mm}$ thickness, $500 \%$ strain rate, alumina fixtures). The moving plate is at the bottom, while the fixed one is at the top. The dotted sine line is an eye guide to visualize the applied shear strain rate. (Courtesy of L. Noirez and U. Windberger, Medical University of Vienna, Austria)

\section{Conclusions}

This entry is motivated by recent experimental results indicating that a first-linear elastic regime exists prior in deformation to the conventional viscoelastic or viscous regime of fluids. The shear elastic regime has been measured at the submillimeter scale in the molten state of various polymers (non-entangled included) pointing out a terminal (low-frequency) solid-like response instead of a flow behavior for frequency range between 0.1 and $10 \mathrm{rad} / \mathrm{s}$. The shear elasticity is a change of paradigm since it means that the flow mechanism is not linked to a molecular process, i.e., to a relaxation time. As a consequence the zero-frequency shear elasticity that imposes the Maxwell model has to be revisited. In polymer physics, the existence of a low-frequency shear elasticity implies that the viscoelastic spectrum cannot be interpreted as the dynamic signature of a single-chain relaxation but should be 
interpreted as a collective response of the material, thus including the intermolecular interactions. We believe that taking into account the liquid cohesion may explain various nonlinear phenomena unpredictable using the conventional single-intrachain approach. The finite shear elasticity means that the flow is obtained at the price of an overpassing an elastic threshold. Correlatively the shear viscosity indicates that the fluid recovers its "static" elasticity after cessation of the constraint. The low-frequency elasticity may explain unpredictable large time scale relaxations, flow-induced instabilities (spurt effect, "shark-skin" instability), shear-induced phases (Pujolle-Robic and Noirez 2001), the correlation between nanoparticles for polymer melt reinforcement (Cassagnau 2003), nonlocal dielectric relaxation (Pronin et al. 2011) or nonuniform temperatures near a solid wall (Noirez et al. 2017), and dynamic heterogeneities (Tracht et al. 1998a, b; Fischer 1983, 2002) involved in the glass process (Brand and Kawasaki 2003).

The consideration of this neglected elastic property enables to predict novel liquid properties as the shear-induced cooling or the establishment of coexisting temperatures under microfluidic conditions. The microfluidic scale is certainly the scale at which the elastic effects are the more visible. The shear elasticity has been mainly detected at the submillimeter scale increasing as the sample dimension decreases. This dimension dependence is coherent with theoretical models predicting static shear elasticity in fluids. On the basis of a survey of various published results, Volino proposed a non-extensive model to describe the liquid shear elasticity and where its dimension dependence is foreseen (Volino 1997). Revisiting the Frenkel model, Trachenko shows the viscous term and the solid-like term can be treated on equal footing in the Maxwell interpolation (Trachenko 2017, Baggioli et al. 2019) Trachenko et al. introduce the concept of local dynamic compressive stress and revisits the Maxwell approach showing that a solid-like approach is also valid by introducing the notion of finite shear wave propagation length (gapped momentum states) (Trachenko et al. 2016; Pronin et al. 2011). These models converge in interpreting the macroscopic liquid behavior as an asymptotic branch of a wider scheme where, at a smaller scale, liquid molecules behave elastically and where the dynamic role of intermolecular interactions is central (Zaccone 2011).

The existence of elastic correlations opens a new route in the approach of the liquid state. The liquid behavior is impacted by more parameters (lengthscale dependence, interfacial force boundaries, compressibilty effects) making its applitive potential richer in particular thermal applications tuning, the flow energy in a shear-induced cooling; and concern the flow mechanisms up to the most confined physiological fluid of the chain of life.

\section{Cross-Reference}

Surface Modifiers for Use with Particulate Fillers

Acknowledgments This work has benefited from the AAP2014 "Instrumentation aux limites" CNRS funding. The author is very pleased to thank her collaborators, Patrick Baroni, Hakima 
Mendil, Philipp, Kahl, Eni Kume and Ursula Windberger. She also would like to thank F. Volino, F. Aitken, D. Aubry, K. Trachenko and A. Zaccone for discussions and theoretical feedback, and R. Ewoldt for stimulating discussion around surface tension. A special thought to the late P.G. de Gennes who chaired the first PhD thesis (www-1lb.cea.fr/theses/mendil_2006.pdf) on the low-frequency shear elasticity in fluids.

\section{References}

Badmaev BB, Bazaron UB, Derjaguin BV, Budaev OR (1983) Measurement of the shear elasticity of polymethylsiloxane liquids. Physica B 122:241-245

Baggioli M, Vasin M, Brazhkin, Trachenko K (2019) Gapped momentum states https://arxiv.org/ abs/1904.01419

Baroni P, Mendil H, Noirez L (2005) French Patent 0510988

Baroni P, Mendil-Jakani H, Noirez L (2010) Innovations pour une mesure complète des propriétés viscoélastiques des fluides. Techniques de l'Ingénieur, TI Ed, 1 RE145

Baroni P, Bouchet P, Noirez L (2013) Highlighting a cooling regime in liquids under submillimeter flows. J Phys Chem Lett 4:2026-2029

Boué F, Bastide J, Buzier M, Collette C, Lapp A, Herz J (1987) Dynamics of permanent and temporary networks: small angle neutron scattering measurements and related remarks on the classical models of rubber deformation. Progr Colloid Polym Sci 75:152-170

Brand HR, Kawasaki K (2003) Are transient positional and orientational order important approaching the glass transition? Physica A 324:484-494

Cassagnau P (2003) Payne effect and shear elasticity of silica-filled polymers in concentrated solutions and in molten state. Polymer 44:2455-2462

Chandler D, Andersen HC (1972) Optimized cluster expansions for classical fluids. II. Theory of molecular liquids. J Chem Phys 57:1930-1931

Chushkin Y, Caronna C, Madsen A (2008) Low-frequency elastic behavior of a supercooled liquid. Euro Phys Lett 83:36001

Collin D, Martinoty P (2002) Dynamic macroscopic heterogeneities in a flexible linear polymer melt. Phys A 320:235-248

Conrad H, Lehmkühler F, Fischer B, Westermeier F, Schroer MA, Chushkin Y, Gutt C, Sprung M, Grübel G (2015) Correlated heterogeneous dynamics in glass-forming polymers. Phys Rev E 91:42309

de Gennes PG (1971) Reptation of a polymer chain in the presence of fixed obstacles. J Chem Phys 55:572-579

Derjaguin BV, Bazaron UB, Zandanova KT, Budaev OR (1989) The complex shear modulus of polymeric and small-molecule liquids. Polymer 30:97-103

Doi M, Edwards SF (1978) Dynamics of concentrated polymer systems. J Chem Soc, Faraday Trans II $74: 1789-1801$

Ferry JD (1970) Viscoelastic properties of polymers. John D. Ferry Wiley, New York, (1961)

Ferry JD (1981) Probing macromolecular motions through viscoelasticity. Rubber Chem Technol 54:76-82

Fischer EW, Bakai A, Patkowski A, Steffen W, Reinhardt L (2002) Heterophase fluctuations in supercooled liquids and polymers. Non-Cryst Solids 307-310:584-601

Fixman M (1991) Stress relaxation in polymer melts and concentrated solutions. J Chem Phys 95:1410-1413

Gallani JL, Hilliou L, Martinoty P, Keller P (1994) Abnormal viscoelastic behavior of side-chain liquid-crystal polymers. Phys Rev Lett 72:2109-2112

Gao J, Weiner JH (1991) Nature of stress on the atomic level in sense polymer in systems of interacting chains. Macromolecules 24:5179-5191

Goyon J, Colin A, Ovarlez G, Ajdari A, Bocquet L (2008) Spatial cooperativity in soft glassy flows. Nature 454:84 
Graham MD (1999) The sharkskin instability of polymer melt flows. Chaos 9:154-163

Guenza M (2002a) Cooperative dynamics in unentangled polymer fluids. Phys Rev Lett 88:025901

Guenza M (2002b) Intermolecular effects in the center-of-mass dynamics of unentangled polymer fluids. Macromolecules 35:2714-2722

Hansen JP, McDonald IR (1991) Theory of simple liquids. Academic Press, London

Hasegawa N, Yuge T, Shimizu A (2016) Liquid is more rigid than solid in a high-frequency region. J Phys Soc Jpn 85:013001

Hatzikiriakos (2012) S.G. wall slip of molten polymers. Prog Polym Sci 37:624-643

Hu HW, Carson GA, Granick S (1991) Relaxation time of confined liquids under shear. Phys Rev Lett 66:2758-2761

Jalocha D, Constantinescu A, Neviere R (2015) Revisiting the identification of generalized Maxwell models from experimental results. Inter J Solid Struct 67-68:169-181

Johnston MT, Ewoldt RH (2013) Precision rheometry: surface tension effects on low-torque measurements in rotational rheometers. J Rheol 57:1515

Kahl P, Baroni P, Noirez L (2013) Hidden solidlike properties in the isotropic phase of the $8 \mathrm{CB}$ liquid crystal. Phys Rev E 88:050501

Kahl P, Baroni P, Noirez L (2015) Harmonic strain-optical response revealed in the isotropic (liquid) phase of liquid crystals. Appl Phys Lett 107:084101

Korolkovas A, Prevost S, Kawecki M, Devishvili A, Adelmann FA, Gutfreund P and Wolf M (2019) The viscoelastic signature underpinning polymer deformation under shear flow, Soft Matter 15:371-380

Demirel LA, Granick S (2001) Origins of solidification when a simple molecular fluid is confined between two plates. J Am Chem Phys 115:1498-1512

Litinov VM, Ries ME, Baughman TW, Henke A, Matloka PP (2013) Chain entanglements in polyethylene melts. Why is it studied again? Macromolecules 46:541-547

Lugorski Karle I, Broakway LO (1944) The structures of biphenyl, o-terphenyl and tetraphenylene. J Am Chem Soc 66:1974-1979

Mansard V, Bocquet L, Colin A (2014) Boundary conditions for soft glassy flows: slippage and surface fluidization. Soft Matter 10:6984-6989

Martinoty P, Hilliou L, Mauzac M, Benguigui L, Collin D (1999) Side-chain liquid-crystal polymers: gel-like behavior below their gelation points. Macromolecules 32:1746-1752

Mendil H (2006) PhD thesis (in french), Paris XI

Mendil H, Grillo I, Baroni P, Noirez L (2006) Frozen states in the isotropic phase of liquid-crystal polymers. Phys Rev Lett 96:077801

Metivier C, Rharbi Y, Magnin A, Bou Abboud A (2012) Stick-slip control of the Carbopol microgels on polymethyl methacrylate transparent smooth walls. Soft Matter 8:7365-7367

Noirez L (2005) Origin of the shear induced transitions. Phys Rev E 72:051701

Noirez L, Baroni P, Mendil-Jakani H (2009a) The missing parameter in rheology: hidden solid-like correlations in liquid polymers and glass formers. Polym Int 58:962-698

Noirez L, Mendil-Jakani H, Baroni P (2009b) New light on old wisdoms on molten polymers: conformation, slippage and shear banding in sheared entangled and unentangled melts. Macromol Rapid Commn 30:1709-1714

Noirez L, Baroni P, (2010) Revealing the solid-like nature of glycerol at ambient temperature. Journal of Molecular Structure 972:16-21

Noirez L, Baroni P, Cao H (2012) Identification of low frequency shear elasticity in liquids n-heptadecane, liquid water and RT-ionic liquids [emim][Tf 2 N]. J Mol Liq 176:71-75

Noirez L, Baroni P, Bardeau JF (2017) Highlighting non-uniform temperatures close to liquid/solid interfaces. Appl Phys Lett 110:213904

Noirez L, Baroni P (2018) Identification of thermal shear bands in a low molecular weight polymer melt under oscillatory strain field. Colloid and Polymer Science 296:713-720

Pronin AA, Trachenko K, Kondrin MV, Lyapin AG, Brazhkin VV (2011) Non local dielectric relaxation in glycerol. Phys Rev B 84:012201 
Pujolle-Robic C, Noirez L (2001) Observation of shear-induced nematic-isotropic transition in sidechain liquid crystal polymers. Nature 409:167-171

Rault J (1987) Space filling and entanglements in polymeric systems. J Non-Newtonian Fluid Mech 23:229-247

Rouse PE (1953) A theory of the linear viscoelastic properties of dilute solutions of coiling polymers. J Chem Phys 21:1272-1280

Scarponi F, Comez L, Fioretto D, Palmieri (2004) Brillouin light scattering from transverse and longitudinal acoustic waves in glycerol. Phys Rev B 70:054203

Sotta P, Deloche B, Herz J, Lapp A, Durand D, Rabadeux JC (1987) Evidence for short-range orientational couplings between chain segments in strained rubbers: a deuterium magnetic resonance investigation. Macromolecules 20:2769-2774

Tolstoi DM (1952) A molecular theory for slippage of liquids over solid surfaces. Dokl Acad Nauk SSSR 85:1089-1092

Trachenko K (2017) Lagrangian formulation and symmetrical description of liquid dynamics. Phys. Rev. E 96:062134.

Trachenko K, Brazhkin VV (2016) Collective modes and thermodynamics of the liquid state. Rep Prog Phys 79:016502-016538

Tracht U, Wilhelm M, Heuer A, Feng H, Schmidt-Rohr K, Spiess HW (1998a) Correlated heterogeneous dynamics in glass-forming polymers. Phys Rev Lett 81:2727

Tracht U, Wilhelm M, Heuer A, Feng H, Schmidt-Rohr K, Spiess HW (1998b) Length scale of dynamic heterogeneities at the glass transition determined by multidimensional nuclear magnetic resonance. Phys Rev Lett 81:2727-2730

Volino F (1997) Théorie viscoélastique non-extensive VI. Application á un liquide formant une phase vitreuse: 1'Ortho TerPhényl (OTP). Ann Phys 22:181-231

Wang S-Q, Ravindranath S, Wang Y, Boukany P (2007) New theoretical considerations in polymer rheology: elastic breakdown of chain entanglement network. J Chem Phys 127:064903

Watanabe H, Kanaya T, Takahashi Y, (2007) Rheo-SANS behavior of Entangled Polymer Chains with Local Label Under Fast Shear Flow, Activity Report on Neutron Scattering Research: Experimental Reports 14:265

Weissenberg K (1948) Proc. 1st Intern. Rheol. Cong. Holland , pp 1-29

Young T (1805) An essay of the cohesion of fluids. Philos Trans Soc Lond 95:65-87

Zaccone A, Blundell JR, Terentjev EM (2011) Network disorder and nonaffine deformations in marginal solids. Phys Rev B 84:174119

Zhu Y, Granick S (2004) Superlubricity: a paradox about confined fluids resolved. Phys Rev Lett 93:096101 